\title{
Functional imaging: A necessary prerequisite to neuropsychological assessment
}

\author{
Edward H Tobe \\ Department of Psychiatry, Cooper Medical School of Rowan University, New Jersey, USA. \\ Correspondence: Edward H Tobe. Address: 1001 Lincoln Dr. W. Suite B, Marlton, NJ 08053-1534, USA. Email: \\ edward.tobe@comcast.net
}

Received: March 24, 2014

Accepted: April 14, $2014 \quad$ Online Published: April 30, 2014

DOI : $10.5430 /$ ijdi.v1n2p74

URL: http://dx.doi.org/10.5430/ijdi.v1n2p74

\section{Abstract}

After traumatic brain injury, neuropsychological testing may be insensitive in documenting functional brain injury. Imaging with single-photon emission computed tomography (SPECT) and ${ }^{18} \mathrm{~F}$-fluorodeoxyglucose positron emission tomography (FDG-PET) scanning may identify brain injury that is missed on neuropsychological testing. A 27-year-old man had loss of consciousness after hydrogen sulfide exposure and a fall; he had markedly impaired function (short-term memory, sequential thinking, attention, and initiative), but neuropsychological testing showed only mild to moderate impairments. A 37-year-old woman had a whiplash injury and head trauma after an automobile accident; she had apprehension, dysphoria, word finding problems, impaired memory and concentration, and slowed thinking; neuropsychological testing was normal. In the man, SPECT scan showed decreased activity in the striatum, amygdala, and hippocampus bilaterally (decreased more in the right than left hemisphere); an FDG-PET scan showed markedly decreased metabolism in the left thalamus, heterogeneous abnormal uptake in the basal ganglia, and abnormally decreased metabolism in both temporal and inferior parietal lobes. In the woman, FDG-PET scan showed several regions of abnormal metabolic activity not restricted to single vascular territories and decreased activity in the left frontal lobe, left thalamus, and left caudate nucleus. Repeat neuropsychiatric testing in both patients showed cognitive and motor impairments that seriously limited routine activities of daily living. In summary, after traumatic brain injury associated with neuropsychological symptoms, SPECT and FDG-PET scanning may be more sensitive than neuropsychological testing in detecting objective signs of brain injury.

\section{Keywords}

Single-photon emission computed tomography, ${ }^{18}$ F-fluorodeoxyglucose, Positron emission tomography, Head trauma, traumatic brain injury, Hydrogen sulfide, Inhalation injury

\section{Introduction}

According to the United States Centers for Disease Control and Prevention, traumatic brain injury is a serious public health problem that affected 2.5 million people in $201{ }^{[1]}$. Traumatic brain injury may cause death and permanent disability. Long-term complications of traumatic brain injury include dementia. Outcome has been linked to prolonged unconsciousness, amnesia, and severity of the initial Glasgow Coma Scale score ${ }^{[2]}$. Most people who sustain one concussion (also known as mild traumatic brain injury) may anticipate full recovery; however, some experience enduring, 
disabling impairments that may include delayed thought processing and cognitive fatigue. Diagnostic accuracy is important for treatment and assessment of prognosis.

Despite the increased sophistication of neuropsychological testing to measure behavioral sequelae of brain injury, there may be discrepancy between the results of these tests and the degree of clinical disability. Mild impairment measured on objective testing may be associated with marked functional impairment of activities of daily living. We hypothesized that functional imaging with single-photon emission computed tomography (SPECT) or ${ }^{18} \mathrm{~F}$-fluorodeoxyglucose positron emission tomography (FDG-PET) scanning may identify brain injury that is missed on neuropsychological testing, but the patients continue to struggle with invalidated cognitive impairment. The present study hopes to illustrate the validity of this hypothesis by reviewing clinical experience with 2 patients who had traumatic brain injury.

\section{Case reports}

\section{Case 1}

A previously healthy 27-year-old male construction worker sustained a work-related injury at a construction site while building a sewer system on a strip of wetland, 500 yards from the ocean. His coworkers were working in a pit (depth, $8 \mathrm{~m}$ ) and were exposed to hydrogen sulfide of unknown concentration. After exposure to the hydrogen sulfide fumes, one coworker died at the scene and another coworker lost consciousness. The patient descended a ladder into the pit in a rescue attempt, but he became affected by the hydrogen sulfide gas and fell an unknown distance. There was no indication that he stopped breathing at any time, but it was believed that he had seizures. He and the unconscious worker were rescued and transported to local hospitals; the other worker was treated with hyperbaric oxygen and released from the hospital within 2 days, and further follow-up was not available.

The patient's past medical history was noncontributory. During grade school, he was a popular, well-adjusted, conscientious student who performed at or above grade level on academic achievement indices. He graduated from a vocational high school, having majored in automotive mechanics (grade point average, 3.0/4). He had received complementary evaluations in categories such as achievement, punctuality, ambition, learning experience, and quality and quantity of work. After graduation he worked in construction. He was well regarded as a designer and builder of recreational vehicles.

After the work injury and upon arrival at the hospital, initial evaluation showed Glasgow Coma Scale score 3, temperature $38.4^{\circ} \mathrm{C}$, heart rate 122 beats per minute, blood pressure 130/65 mm Hg, and respiratory rate 16 breaths/minute. He had no skin abrasions or bruising on the head. He had seizure activity and decerebrate posturing, and pupils were dilated and sluggish in response to light. No spontaneous movements were noted, corneal reflexes were absent, and deep tendon reflexes were 2/4. Blood carboxyhemoglobin level was 0.15. Electrocardiogram showed sinus tachycardia. Computed tomography (CT) of the chest showed pulmonary edema, but CT of the head was normal.

At 10 hours after hydrogen sulfide exposure, the patient received a dose of sodium nitrite (300 $\mathrm{mg}$ ), furosemide (20 mg), and vecuronium (10 mg), and started hyperbaric oxygen treatments (29.4 psi for 45 minutes, twice daily). On day 5 after admission, he regained consciousness and responded to simple commands. On day 7 after admission, the Glasgow Coma Scale score was 15 and hyperbaric oxygen treatments were discontinued. He could feed himself and walk with assistance; but, he appeared agitated and restless and demonstrated impaired language, memory, and attention.

At 16 days after hospital admission, he was transferred to a rehabilitation facility. He had slow speech, impaired attention span, retrograde amnesia with confabulation, decreased insight and ability to communicate, flat affect, and impaired visual memory for acquisition, retention, and recall of new information. At discharge from the rehabilitation facility, one month after injury, neuropsychological evaluation showed difficulty on verbal and visual memory tasks and associative learning. 
He required cues to perform simple jobs including rudimentary planning, organizing, and sequencing. He had left-sided body deficits in balance and speed of movement. At 17 months after injury, magnetic resonance imaging (MRI) of the brain showed no abnormal signal, mass, midline shift, or sulcal effacement, and the posterior fossa, base of the skull, and all visible structures appeared normal.

During a four year time interval after the accident, he had persistent impairment of short-term memory, sequential thinking, attention, and initiative. He remained unemployable, had no initiative to perform routine household chores, and was oblivious about his disabilities.

At 2 years after the injury, neuropsychological testing (Halstead-Reitan Neuropsychological Test Battery; Wechsler Memory Scale - Revised; Wechsler Adult Intelligence Scale - Revised; and Rey-Osterrieth Complex Figure Test) showed mild to moderate impairments (memory and attention, common sense reasoning, social judgment, verbal concept formation, and abstract reasoning). The neuropsychological diagnosis was mild encephalopathy and prognosis was rated fair to good.

At 3 years after the injury, a cerebral perfusion study with SPECT scanning of the brain showed decreased activity in the striatum, amygdala, and hippocampus bilaterally (decreased more in the right than left hemisphere) (see Figure 1). Brain MRI scan 1 month later was normal, with no lesions in the basal ganglia. An FDG-PET scan at 3 years after the accident showed markedly decreased metabolism in the left thalamus, heterogeneous abnormal uptake in the basal ganglia, and abnormally decreased metabolism in both temporal and inferior parietal lobe.
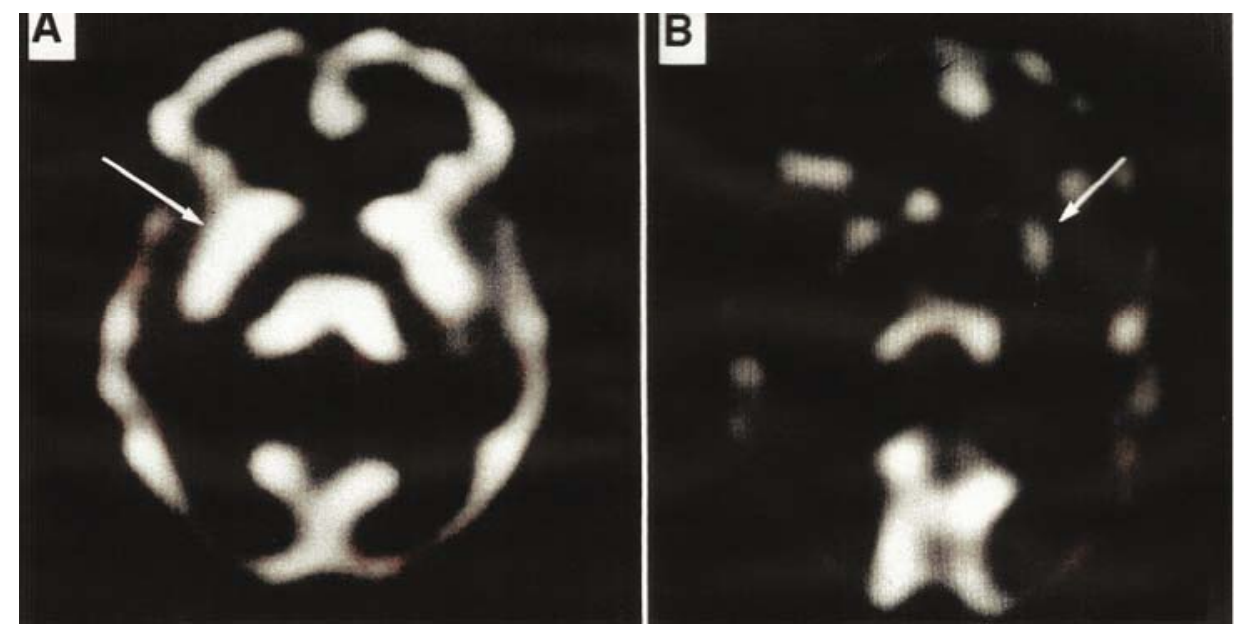

Figure 1. A 30-Year-Old Man 3 Years After Hydrogen Sulfide Exposure and Unconsciousness (Case 1). Single-photon emission computed tomography (SPECT) images of the brain were acquired at 1 hour after intravenous administration of hexamethylpropylene amine oxime labeled with technetium Tc 99m (740 MBq [20 mCi]) (Ceretec, Amersham International, Arlington Heights, IL, USA) on a triple-headed camera equipped with fan beam collimators (Picker International, Cleveland, OH, USA). The intrinsic resolution of the camera was rated at $8 \mathrm{~mm}$ to $9 \mathrm{~mm}$ full width half maximum. The back projected images were reconstructed with a count-rate-dependent restoration filter. The modulation transfer function was generated from the line-spread function of the camera. Chang method was used to correct the SPECT images for attenuation with a uniform ellipse. The findings were assessed with reference to a normal database of 44 volunteers. (A) The patient had decreased activity in the striatum (arrow) amygdala, and hippocampus bilaterally (decreased more in the right than left hemisphere). (B) Reference scan from a neurologically intact person.

At 4 years after injury, additional neuropsychological testing for motor function (Unified Parkinson Disease Rating Scale; Cambridge Automated Neuropsychological Test Assessment Battery), cognitive function mediated by basal ganglia, and executive function (Tower of Hanoi Planning Test) showed slowing of movement (an isolated extrapyramidal sign), 
slowing of central information processing, and severe impairment of spatial memory, visual working memory, and executive function. The test results were interpreted as evidence that the cognitive and motor impairments seriously limited his ability to perform routine activities of daily living and that improvement was unlikely.

\section{Case 2}

A 37-year-old woman was injured in an automobile accident. She was driving a van and was broadsided on the passenger side. She recalled that her van lifted slightly and bounced down. Her head whipped to one side and then back, striking the head rest. She did not know whether she lost consciousness. She recalled feeling frightened, dizzy, lightheaded, hot, and nauseous, and as if she were "floating in a cloud" for several hours after the accident. She drove the partially disabled vehicle to her house and reported that she began to feel pain the next day. Past medical history was noncontributory, with no previous history of chronic medical problem or previous head trauma. She was a high school graduate, was married, had 2 adolescent children, worked part-time as a school aid, and was functioning well before the accident. She did not smoke and she drank a glass of wine occasionally at dinner.

At initial evaluation, 30 months after the automobile accident, she reported nervousness, depression, disrupted sleep, fatigue, and recurrent headaches. She described memory problems, especially verbal memory such as reading recall and word finding. She reported impaired attention concentration and that she felt "stupid." The mental status examination showed apprehension, dysphoria, word finding problems, impaired memory and concentration, and slowed thinking. She required repeated assurance that what she said was clear. The physical and neurological examinations were normal. A brain MRI scan was normal.

At 3 years after the injury she had ongoing memory complaints. Neuropsychological tests (Halstead-Reitan Neuropsychological Test Battery; Wechsler Memory Scale - Revised; Wechsler Adult Intelligence Scale - Revised; and Minnesota Multiphasic Personality Inventory) were normal, with no evidence of any organic brain dysfunction. Her symptoms were interpreted as being caused by depression. Psychopharmacologic treatment brought the depression into remission but she continued to experience cognitive problems. After the neuropsychological testing, an FDG-PET scan showed several regions of abnormal metabolic activity not restricted to single vascular territories and decreased activity in the left frontal lobe, left thalamus, and left caudate nucleus (see Figure 2).

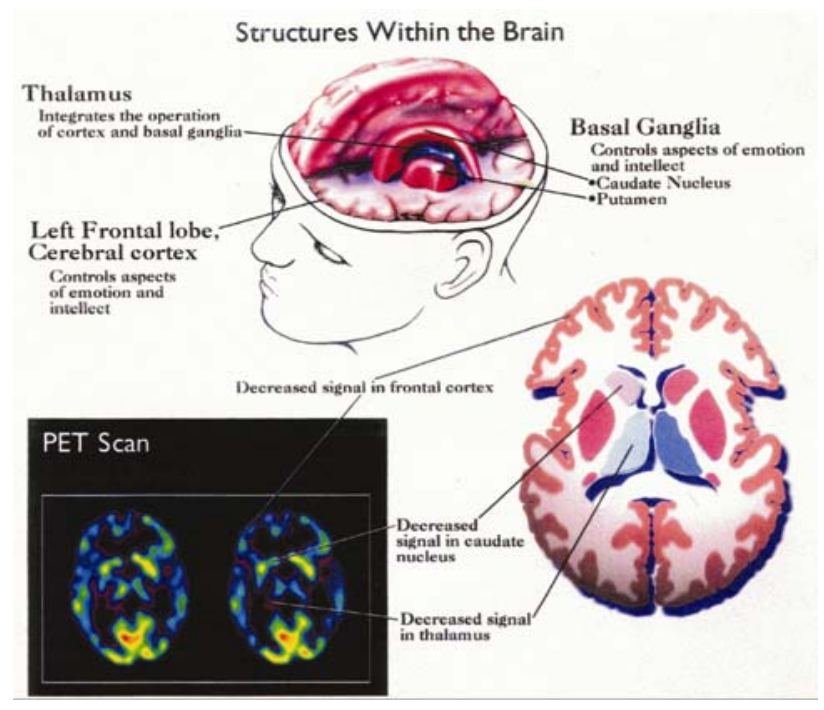

Figure 2. A 40-Year-Old Woman 3 Years After An Automobile Accident. The ${ }^{18}$ F-fluorodeoxyglucose positron emission tomography (FDG-PET) scan showed several regions of abnormal metabolic activity not restricted to single vascular territories and decreased activity in the left frontal lobe, left thalamus, and left caudate nucleus. 
At 5 years after injury, additional neuropsychological testing for motor function (Unified Parkinson Disease Rating Scale; Cambridge Automated Neuropsychological Test Assessment Battery); cognitive functions mediated by the basal ganglia; and attention (Stroop Color-Word Test) showed slowing of movement (an isolated extrapyramidal sign); slowing of essential information processing; severe impairment of spatial memory and visual working memory; and severe impairment of attention. The test results were interpreted as evidence that the cognitive and motor impairments seriously limited her ability to perform routine activities of daily living and that improvement was unlikely.

\section{Discussion}

In these patients, neuropsychological testing before imaging showed normal results or mild to moderate impairment, and prognosis initially was believed favorable. However, subsequent functional imaging gave objective evidence of marked brain damage. Subsequent repeat neuropsychological testing showed severe impairment of function and prognosis was poor in both patients. These cases demonstrate the value of functional imaging in the evaluation of neuropsychological impairment after inhalation injury and head trauma, despite normal or mildly abnormal neuropsychological tests.

The FDG-PET imaging to study brain metabolism is based on mitochondrial respiration that requires glucose, but neurons do not store glucose. Glucose is transported across the plasma membrane of the cell by a glucose transporter protein (GLUT) that has several isoforms in different tissues. The isoform GLUT1 transports glucose and other hexose molecules across the blood-brain barrier and astrocyte plasma membrane, and the isoform GLUT3 transports glucose into the neuron ${ }^{[3]}$. The FDG-PET scan uses FDG, an analog of glucose, that emits positrons when glucose is metabolized within the cell. The brain regulates its blood supply according to metabolic need. The SPECT images may evaluate brain metabolism using radiopharmaceuticals that emit gamma rays ${ }^{[4]}$.

In both patients, initial neuropsychological testing underestimated or missed clinical pathology. As a result of missed pathology, insurance carriers, health care providers, and family members may question the authenticity of the patient's complaints. Functional imaging provided objective guidance to select suitable, focused neuropsychological evaluation to demonstrate otherwise undiscovered pathology. Functional imaging may prove an important guide before Neuropsychological testing.

\section{References}

[1] Centers for Disease Control and Prevention. Traumatic brain injury. [Internet] Available from: http://www.cdc.gov/TraumaticBrainInjury/index.html. Accessed March 15, 2014.

[2] Sayed, N., Culver, C., Dams-O'Connor, K., Hammond, F., \& Diaz-Arrastia, R. Clinical phenotype of dementia after traumatic brain injury. J Neurotrauma. 2013; 30(13): 1117-1122. http://dx.doi.org/10.1089/neu.2012.2638

[3] Gould, G.W., \& Holman, G.D. The glucose transporter family: structure, function and tissue-specific expression. Biochem J. 1993; 295(Pt 2): 329-341.

[4] Tobe, E.H. Mitochondrial dysfunction, oxidative stress, and major depressive disorder. Neuropsychiatr Dis Treat. 2013; 9: 567-573. http://dx.doi.org/10.2147/NDT.S44282. 